\title{
Early cognitive basic symptoms are accompanied by neurocognitive impairment in patients with an 'at-risk mental state' for psychosis
}

\author{
Sarah Eisenacher, ${ }^{1 *}$ Franziska Rausch, ${ }^{1}$ * Fabian Ainser, ${ }^{1}$ Susanne Englisch, ${ }^{1}$ Anna Becker, ${ }^{1}$ Daniela Mier, ${ }^{2}$ \\ Sabrina Fenske, ${ }^{2}$ Andreas Meyer-Lindenberg, ${ }^{1}$ Peter Kirsch $^{2}$ and Mathias Zink ${ }^{1}$
}

\begin{abstract}
${ }^{1}$ Department of Psychiatry and Psychotherapy, and ${ }^{2}$ Department of Clinical Psychology, Central Institute of Mental Health, Medical Faculty Mannheim, University of Heidelberg, Heidelberg, Germany

\section{Corresponding author: Mrs. Sarah Eisenacher, Central Institute of Mental Health, Department of Psychiatry and Mannheim/Heidelberg University, Square J5, 68159 Mannheim, Germany. Email: sarah.eisenacher@zi-mannheim.de} Psychotherapy, Medical Faculty
\end{abstract}

Conflicts of Interest: Sarah Eisenacher, Franziska Rausch, Fabian Ainser, Anna Becker, Daniela Mier, Sabrina Fenske and Peter Kirsch: NO COI.Susanne Englisch has received travel expenses and consultant fees from AstraZeneca, Bristol-Myers Squibb GmbH \& CoKGaA, Eli-Lilly, Janssen Cilag, Otsuka Pharma, Pfizer Pharma and Servier. Andreas Meyer-Lindenberg receives consultant fees and travel expenses from AstraZeneca, Hoffmann-La Roche, Lundbeck Foundation. Speaker's fees from Pfizer Pharma, Lilly Deutschland, Glaxo SmithKline, Janssen Cilag, BristolMyers Squibb, Lundbeck, Servier and AstraZeneca.Mathias Zink received unrestricted scientific grants of the European Research Advisory Board (ERAB), German Research Foundation (DFG), Pfizer Pharma GmbH, Servier and Bristol Myers Squibb Pharmaceuticals; further speaker and travel grants were provided from Astra Zeneca, Lilly, Pfizer Pharma GmbH, Bristol Myers Squibb Pharmaceuticals, Otsuka, Servier Lundbeck, Janssen Cilag, Roche and Trommsdorff.

*Both first authors contributed equally

\begin{abstract}
Aim: Patients with an increased risk for psychosis ('at-risk mental state' (ARMS)) present various neurocognitive deficits. Not least because of differences in identifying the ARMS, results of previous studies are inconsistent. In most studies ARMS-patients are classified by the experience of attenuated psychotic symptoms (APS) and/or brief limited intermittent psychotic symptoms (BLIPS). Few studies additionally assessed cognitive basic symptoms (BS). A comprehensive assessment in the very early stage of the ARMS is missing.
\end{abstract}

Methods: In the present study we characterized ARMS-patients for cognitive BS (ARMS-BS), APS and BLIPS (ARMS-A/B) according to the Early Recognition Inventory based on IRAOS (ERIraos). Furthermore, we assessed neurocognitive deficits using the MATRICS consensus cognitive battery for schizophrenia with a primary hypothesis regarding working memory performance. Groups of 38 ARMS-

Key words: at-risk mental state, cognition, ERIraos, prodrome, working memory. matched for age, gender, education and premorbid verbal intelligence.

Results: Between-group comparisons revealed significant poorer working memory performance in addition to lower verbal learning and problem solving, slower processing speed and lower global neurocognitive functioning in ARMS-patients as compared to controls. ARMS-BS did not differ from ARMS-A/B.

Conclusions: These results underscore the presence of cognitive limitations in patients only presenting with cognitive BS. Knowledge of these early cognitive deviations supports the inclusion of early ARMS-stages into a comprehensive concept of the psychosis risk state. Therapeutic interventions already applied at this stage might prevent deterioration of constraints. Longitudinal and interventional studies investigating the interaction of cognitive BS and neurocognitive as well as metacognitive deficits are warranted. patients and 38 healthy controls were 


\section{INTRODUCTION}

Schizophrenia patients typically display psychotic positive, as well as negative symptoms and a broad range of neurocognitive deficits. ${ }^{1}$ These determine the prognosis of social and vocational rehabilitation to a large extent. ${ }^{2,3}$ Neurocognitive deficits mainly concern the domains attention and vigilance, executive functioning and problem solving, working memory, processing speed as well as verbal and visual learning. ${ }^{4}$ Among these domains, working memory impairment has been found to be a central feature of schizophrenia. ${ }^{5}$ This impairment is even discussed as an endophenotypic marker of the illness ${ }^{6}$ as it has been consistently found in patients with schizophrenia $^{7}$ and unaffected relatives. ${ }^{8}$ Furthermore, it seems to be state independent ${ }^{9}$ and specific to schizophrenia spectrum disorders. ${ }^{6}$

Several prior studies have reported findings about neurocognitive deficits in the 'at-risk mental state' (ARMS) for psychosis, e.g. in working memory, verbal memory, executive control and processing speed. ${ }^{10-18}$ These results are summarized in a recent meta-analysis ${ }^{19}$ which states that neurocognitive deficits are evident before the first onset of psychosis and do not decline over a 5-year period of illness. Compatible with findings in patients with schizophrenia, working memory impairment has been discussed to be one core neurocognitive deficit in the ARMS, differentiating between those patients with and without a transition to psychosis. ${ }^{20}$ Visuospatial ${ }^{21-23}$ as well as verbal working memory abilities $^{13,24,25}$ seem to be affected. Furthermore, processing speed deficits form an important dimension within the pathogenesis of psychosis. Poor performance in an ultra-high risk community sample ${ }^{26}$ in a birth cohort study ${ }^{27}$ and in adolescents who later developed a schizophreniform disorder ${ }^{28}$ propose a potential predicting function of psychotic experiences. Supporting these findings, a longitudinal study showed that the neurocognitive performance of ARMS-patients who later experienced a transition to psychosis only deteriorated in the domains working memory and processing speed over a 6 -months course. ${ }^{14}$

Most studies classify ARMS-patients by 'ultrahigh risk' (UHR)-criteria for schizophrenia spectrum disorders, diagnosed by the presence of attenuated psychotic symptoms (APS) and/or brief limited intermittent psychotic symptoms (BLIPS) or by a genetic risk and a deterioration syndrome. ${ }^{29,30}$ These symptoms are generally assessed by the 'Structured Interview for Prodromal Syndromes' (SIPS) ${ }^{31}$ including the 'Criteria Of Prodromal Syndromes' (COPS) or the
'Comprehensive Assessment of At Risk Mental States' (CAARMS). ${ }^{32}$ Hence, the majority of reviews and meta-analyses on neurocognitive deficits in the ARMS mainly include studies defining the ARMS according to SIPS/SOPS or CAARMS. ${ }^{11,19,33,34}$

However, Huber, Gross and Klosterkötter described cognitive basic symptoms (BS) ${ }^{35}$ that already occur in early ARMS-stages. ${ }^{36,37}$ They thereby added an important symptom cluster which the UHR-definition does not account for. Cognitive BS display the core symptoms of the 'Bonn Scale for the Assessment of Basic Symptoms' (BSABS) ${ }^{35}$ and the 'Schizophrenia Proneness Instrument - Adult version' (SPI-A) ${ }^{38}$ and are also represented in the ERIraos (Early Recognition Inventory for the Retrospective Assessment of the Onset of Schizophrenia based on IRAOS $)^{39-41}$ ERIraos aims at the feasible detection of an increased risk for psychosis by concentrating on the presence of symptoms during the last 12 months, and provides a detailed assessment of APS, BLIPS and cognitive BS within one scale. In comparison to the CAARMS, ERIraos has been demonstrated to be equally sensitive in the detection of APS and BLIPS. With the inclusion of cognitive BS it further allows for a high sensitivity in the assessment of the whole spectrum of ARMS-symptoms and thereby reduces false-negative attributions. ${ }^{41}$ In summary, as cognitive BS have become increasingly important in prodromal research, ${ }^{18,37,42}$ it seems crucial to integrate them into the identification of ARMSpatients when assessing neurocognitive deficits. So far, relatively few studies assessed cognitive BS when analyzing neurocognitive performance in the ARMS and reported inconsistent results. ${ }^{10,13,43,44}$ Furthermore different scales were used in these studies in order to assess the whole spectrum of symptomatology.

The aim of the present study was to assess neurocognitive deficits in an ARMS-sample that was characterized by the final version of the ERIraos, focusing on cognitive BS in addition to APS and BLIPS (see methods for details). Our core hypothesis was that ARMS-patients characterized by ERIraos display a deficit in the domain working memory as well as in the two subtests of verbal and spatial working memory. Furthermore, we assumed to find a deficit regarding global ('composite') neurocognitive functioning. Secondary endpoints included between-group comparisons of further cognitive domains, between-group comparisons of ARMSsubsamples (patients with cognitive BS only 
(ARMS-BS) vs. patients with APS and/or BLIPS (ARMS-A/B)), and finally a description of the interplay of cognitive BS and neurocognitive deficits.

\section{METHODS}

\section{Participants}

The present study was approved by the local ethics committee of the Medical Faculty Mannheim of the University of Heidelberg (AZ 2009-296N-MA). Patients met the pre-defined inclusion criteria: (i) attribution to ARMS according to ERIraos ${ }^{39-41}$ (sum score $\geq 30$ and/or presence of at least two cognitive BS and/or at least one APS and/or at least one BLIPS); (ii) age between 18 and 40 years; (iii) ability to provide written informed consent; and (iv) sufficient German language skills. Patients who fulfilled the criteria for a first episode of psychosis, for substance dependence excluding nicotine, or for other disorders of the central nervous system requiring treatment were excluded. A number of 38 ARMS-patients were included into the study. None of them were treated with antipsychotics. Stable pre-medication with antidepressants (citalopram $(n=2)$, mirtazapine $(n=2)$, sertraline $(n=2)$, paroxetine $(n=1)$, duloxetine $(n=1)$, trimipramine $(n=2))$ was allowed. Because of anxiety or agitation four patients were treated with low doses of lorazepam. Three patients had a positive family history of schizophrenia in first degree relatives and further six patients in second degree relatives. A number of 16 patients reported cannabis abuse in the past. Six of them indicated intermittent or one-time abuse of further substances (hallucinogens $=2$, amphetamine $=5$, cocaine $=3$ ). In any case, last drug use was at least four weeks prior to study entry. None of them fulfilled the criteria for drug addiction. A number of 38 healthy control subjects (HC) were matched for gender, age, levels of education and premorbid verbal intelligence (Table 1), had no positive family history of schizophrenia, bipolar disorder or suicide in first-degree relatives, and did neither have any previous or current psychiatric disorders according to the M.I.N.I. (Mini-International Neuropsychiatric Interview) nor any former or present psychopharmacological treatment.

\section{Psychometric rating scales}

ARMS-symptoms and general psychopathology were characterized by trained and certified clinicians using ERIraos, PANSS (Positive and
Negative Syndrome Scale) and PSYRATS (Psychotic Symptoms Rating Scale). PANSS composite scores ${ }^{45}$ as well as the PANSS five factor-model ${ }^{46}$ were evaluated. We further assessed negative symptoms (Scale for the Assessment of Negative Symptoms: SANS), comorbid depressive symptoms (Calgary Depression Scale for Schizophrenia: CDSS), general severity of illness (Clinical Global Impression: CGI) and psychosocial functioning (Global Assessment of Functioning: GAF; Personal and Social Performance Scale: PSP).

\section{Neurocognitive characterization}

Cognitive abilities were assessed using the MATRICS consensus cognitive battery (MCCB) for schizophrenia, ${ }^{47}$ that includes 10 tests representing seven domains, namely working memory (Letter-Number Sequencing, Spatial Span), verbal (Hopkins Verbal Learning Task revised) and visual learning (Brief Visuo-Spatial Memory Test revised), speed of processing (Trail Making Test version A, Symbol Coding, Verbal Fluency), problem solving (Mazes), social cognition (Meyer-Salovey-Caruso Emotional Intelligence Test), and vigilance (Continuous Performance Test - identical pairs). Additionally, attention and executive functioning were assessed using the Trail Making Test, version B (TMT-B) and the Wisconsin Card Sorting Test (WCST). Estimated premorbid verbal intelligence was assessed using the Multiple Choice Word Test, version B (MWT-B).

\section{Statistical evaluation}

Based on existing data, ${ }^{48}$ we estimated the required sample size to detect group differences using the statistical software $\mathrm{G}^{*}$ power 3.1. ${ }^{49}$ Given a significance level of 0.05 and an effect size of $d=0.74$, at least 24 participants per group were considered sufficient for achieving a power of 0.8. Statistical analyses were conducted using the Statistical Package for Social Sciences (IBM SPSS version 20.0, Chicago, IL, US). Neurocognitive performance was expressed in terms of standardized $t$-values. Single tests representing the same domain contributed equally to domain scores. Furthermore, a global composite score was calculated, also indicated by a $t$-value. ${ }^{50}$ Descriptive statistics included means and standard deviations. We tested for non-normal distributions of parameters using histograms and the Kolmogorov-Smirnov test. Scores of two domains were slightly skewed to the right, namely problem solving within the control group and 
TABLE 1. Socio-demographic and psychopathological characteristics of study samples

\begin{tabular}{|c|c|c|c|}
\hline & Patients $(n=38)$ & Controls $(n=38)$ & Comparison \\
\hline \multicolumn{4}{|l|}{ Socio-demographics } \\
\hline Age & $22.9 \pm 4.17$ & $24.3 \pm 5.78$ & $t(74)=-1.206, p=0.232$ \\
\hline Gender (female/male) & $13 / 25$ & $12 / 26$ & Fisher exact: $X^{2}(1)=.06 p=1.000$ \\
\hline $\begin{array}{l}\text { School years } \\
\text { MWT-B }\end{array}$ & $11.2 \pm 1.56$ & $11.6 \pm 1.48$ & $t(74)=-1.208, p=0.231$ \\
\hline Raw score & $23.9 \pm 4.70$ & $24.0 \pm 5.72$ & $t(74)=-0.110, p=0.913$ \\
\hline $\begin{array}{l}\text { Estimated verbal IQ } \\
\text { ERIraos }\end{array}$ & $96.2 \pm 10.67$ & $97.5 \pm 13.27$ & $t(74)=-0.448, p=0.656$ \\
\hline Sum score & $42.8 \pm 15.61$ & n.a. & n.a. \\
\hline $\begin{array}{l}\text { Early ARMS/late ARMS } \\
\text { PANSS }\end{array}$ & $11 / 27$ & n.a. & n.a. \\
\hline Total score & $61.3 \pm 11.88$ & n.a. & n.a. \\
\hline Positive symptoms & $12.8 \pm 4.06$ & n.a. & n.a. \\
\hline Negative symptoms & $13.5 \pm 5.17$ & n.a. & n.a. \\
\hline $\begin{array}{l}\text { Global psychopathology } \\
\text { Additional psychometric scales }\end{array}$ & $35.1 \pm 6.83$ & n.a. & n.a. \\
\hline SANS & $31.2 \pm 21.38$ & n.a. & n.a. \\
\hline CDSS & $6.7 \pm 4.32$ & n.a. & n.a. \\
\hline PSP & $57.5 \pm 15.76$ & n.a. & n.a. \\
\hline GAF & $48.5 \pm 10.77$ & n.a. & n.a. \\
\hline $\begin{array}{l}\text { CGI-Severity } \\
\text { PSYRATS }\end{array}$ & $4.1 \pm 0.56$ & n.a. & n.a. \\
\hline Conviction & $1.3 \pm 1.51$ & n.a. & n.a. \\
\hline Sum score & $8.18 \pm 7.9$ & n.a. & n.a. \\
\hline
\end{tabular}

Data is reported as mean \pm standard deviation (SD) or frequencies. Abbreviations: ARMS, At-Risk Mental State; CDSS, Calgary Depression Scale for Schizophrenia; CGI, Clinical Global Impression; ERIraos, Early Recognition Inventory based on IRAOS; GAF, Global Assessment of Functioning; IQ, intelligence quotient; IRAOS, Interview for the Retrospective Assessment of the Onset of Schizophrenia and Other Psychoses; MWT-B, Multiple Choice Word Test (version B); n.a., not applicable; PANSS, Positive and Negative Syndrome Scale; PSP, Personal and Social Performance Scale; PSYRATS, Psychotic Symptoms Rating Scale; SANS, Scale for the Assessment of Negative Symptoms.

visual learning within both groups. Between-group comparisons were performed with Student's $t$-test or univariate analyses of variance. Because group sizes were equal we assumed a robustness of these tests against non-normality. ${ }^{51,52}$ In a second groupcomparison, all $t$-values were transformed into dichotomous variables to either represent deficient $(t$-value $<40)$ or normal-range $(t$-value $\geq 40)$ scores. These were compared using Fisher's Exact tests.
Correlations were analysed with Pearson`s correlation coefficient $(r)$ or Spearman rank correlations. Linear regression analyses were performed using the 'Enter' method to analyse the interplay between ARMS symptoms and neuropsychological performance. Because of the number of comparisons, Bonferronicorrection for multiple testing was applied for the exploratory data analysis, which included all further MCCB domains. 


\section{RESULTS}

\section{Participants}

Socio-demographic and psychometric data are provided in Table 1. There were no significant differences regarding age, gender, education and premorbid verbal intelligence. Patients were characterized as moderately affected according to PANSS, PSYRATS and ERIraos. The mean ERIraos sum score was $42.8 \pm 15.61$. Eleven patients were attributed to ARMS-BS and 27 patients to ARMS-A/B.

\section{Between group comparisons}

Regarding working memory, ARMS-patients $(49.34 \pm 9.10)$ displayed a significant deficit compared to HCs $(55.18 \pm 10.04 ; T=-2.66, d f=74$, $P=0.010, \quad 95 \% \quad$ CI $[-10.22,-1.46], \quad d=0.610)$. Regarding the differentiation between spatial and verbal working memory, ARMS-patients performed significantly worse in the spatial span-task $(50.39 \pm 10.04$ vs. $57.16 \pm 9.80, T=2.97$, $d f=74, P=0.004,95 \%$ CI $[2.23,11.30], d=0.682)$, but no significant difference emerged for the letter-number-sequencing ( $48.32 \pm 8.16$ vs. 51.26 $\pm 10.17, T=1.39, d f=74, P=0.168,95 \%$ CI $[-1.27$, 7.16], $d=0.320$ ). Furthermore, a between group comparison of global ('composite') neurocognitive functioning revealed a significant lower performance in ARMS-patients (ARMS: $43.3 \pm 8.69$ vs. HCs: $51.1 \pm 9.37 ; T=-3.76, d f=74$, $p<0.001,95 \%$ CI $[-11.92,-3.66], d=0.86)$ ( Table 2).

ARMS-patients and HCs were further compared regarding additional MATRICS domains (Fig. 1) as well as TMT-B and WCST. After correction for multiple testing we observed significant group differences regarding processing speed, verbal learning and problem solving (Table 2). A comparison

TABLE 2. Neurocognitive characteristics of study samples

\begin{tabular}{|c|c|c|c|}
\hline & Patients $(n=38)$ & Controls $(n=38)$ & Comparison \\
\hline \multicolumn{4}{|l|}{ MATRICS test battery } \\
\hline Working Memory (LNS, Spatial Span) & $49.34 \pm 9.10$ & $55.18 \pm 10.04$ & $T(74)=-2.66, p=0.010$ \\
\hline Processing Speed (TMT-A, BACS-SC, Fluency) & $44.24 \pm 8.68$ & $50.24 \pm 10.87$ & $F(1,74)=7.072, p=0.010$ \\
\hline Verbal Learning(HVLT-R) & $51.13 \pm 10.98$ & $58.53 \pm 12.40$ & $F(1,74)=7.572, p=0.007$ \\
\hline Visual Learning (BVMT-R) & $47.42 \pm 10.79$ & $50.42 \pm 8.05$ & $F(1,74)=1.837, p=.174$ \\
\hline Planning and Problem Solving (NAB-Mazes) & $46.16 \pm 8.70$ & $51.76 \pm 6.85$ & $F(1,74)=9.743, p=0.003$ \\
\hline Vigilance (CPT-IP) & $39.53 \pm 9.02$ & $42.82 \pm 9.02$ & $F(1,74)=2.528, p=.116$ \\
\hline Social Cognition (MSCEIT) & $43.84 \pm 10.45$ & $46.71 \pm 9.15$ & $F(1,74)=1.622, p=.207$ \\
\hline Global Composite & $43.34 \pm 8.69$ & $51.13 \pm 9.37$ & $T(74)=-3.759, p<0.001$ \\
\hline TMT-B (sec) & $59.42 \pm 17.34$ & $56.84 \pm 17.82$ & $F(1,74)=0.409 p=0.525$ \\
\hline \multicolumn{4}{|l|}{ WCST } \\
\hline Total Errors (\%) & $20.15 \pm 7.33$ & $18.33 \pm 5.73$ & $F(1,74)=1.457, p=0.231$ \\
\hline Perseveration Score (\%) & $17.75 \pm 13.95$ & $16.55 \pm 15.42$ & $F(1,74)=0.127, p=0.723$ \\
\hline Concept perseverations & $0.76 \pm 1.15$ & $0.53 \pm 0.89$ & $F(1,74)=1.007, p=0.319$ \\
\hline Failure to maintain set & $0.79 \pm 1.28$ & $0.76 \pm 1.32$ & $F(1,74)=0.008, p=0.930$ \\
\hline
\end{tabular}

Data is reported as mean \pm standard deviation (SD). Domain scores of the MATRICS domains are reported as standardized $t$-values. Bonferroni correction for multiple testing was applied for all MATRICS domains except 'Working Memory' and 'Global Composite', because these were tested as separate core hypotheses. Abbreviations: BACS-SC, Brief Assessment of Cognition in Schizophrenia, Symbol Coding; BVMT-R, Brief Visual Memory Test Revised; CPT-IP, Continuous Performance Test, Identical Pairs; HVLT-R, Hopkins Verbal Learning Task Revised; LNS, Letter-Number-Sequencing; MATRICS, Measurement and Treatment Research to Improve Cognition in Schizophrenia; MSCEIT, Meyer-Salovey-Caruso Emotional Intelligence Test; NAB, Neuropsychological Assessment Battery; n.s., not significant; sec, seconds; TMT-A or -B, Trail Making Test (version A and B); WCST, Wisconsin Card Sorting Test. 
FIGURE 1. Means of standardized $t$-values of the MATRICS domains. Bonferroni correction for multiple testing was applied for all MATRICS domains except 'Working Memory' and 'Global Composite', because these were tested as separate hypotheses. Abbreviations: ARMS, At-Risk Mental State; AttVig, Attention and vigilance; Comp, global ('composite') neurocognitive functioning; MATRICS, Measurement and Treatment Research to Improve Cognition in Schizophrenia; PrblmS, Problem Solving; SclCgn, Social Cognition; SpdPr, Processing speed; VisL, Visual Learning; VrblL, Verbal Learning; WM, Working memory; * significant, a ARMS and $\square$ Controls.

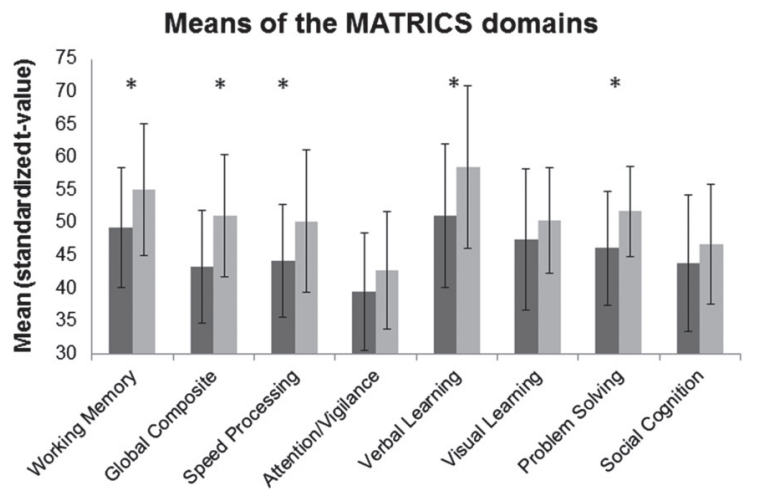

of the frequencies of deficits revealed no significant results. There was a trend visible for problem solving $(\mathrm{X}(1, n=76)=4.145, P=.086)$.

Within the secondary endpoints, we stratified the ARMS-group into the subgroups ARMS-A/B (with $(n=20)$ or without $(n=7)$ coexisting BS) or ARMS-BS ( $n=11$, without any APS or BLIPS). Univariate analyses of variance revealed significant groupdifferences for working memory (HC: $55.18 \pm 10.04$, BS: $48.91 \pm 10.26$, APS/BLIPS: $49.52 \pm 8.79 ; \quad F(2,73)$ $=3.5, P=0.035, \eta_{p}{ }^{2}=.087$ ) and for global ('composite') neurocognitive functioning (HC: $51.13 \pm 9.37$, BS: $42.27 \pm 10.60, \quad$ APS/BLIPS: $43.78 \pm 7.96 ; \quad F \quad(2,73)$ $\left.=7.096, P=0.002, \eta_{p}{ }^{2}=.163\right)$. Post-hoc comparisons (least square differences) revealed a trend towards significance between controls and ARMS-BS $(P=.061,95 \%$ CI $[-0.31,12.86])$ in working memory performance, but no difference between ARMS-BS and ARMS-A/B $(P=.860,95 \%$ CI $[-7.98,4.97])$. Regarding the global neurocognitive functioning, HCs significantly differed from ARMS-BS $(P=.006$, 95\% CI [2.66, 15.06]), but again no differences between ARMS-BS and ARMS-A/B became apparent $(P=.645,95 \%$ CI $[-7.98,4.97])$. Because the ARMSBS group was rather small, we added chi-squared tests and compared the three experimental groups according to their working memory and global neurocognition performance, both categorized by a median split. The results were significant for working memory (working memory: $\mathrm{X}^{2}(2, \quad n=76)=7.877$,
$P=.019$, Cramer's $V=.322$; composite score: $X^{2}(2$, $n=76)=4.455, P=.108$, Cramer's $V=.242$ ). Further analyses revealed a significant difference between $\mathrm{HC}$ and ARMS-BS $\left(\mathrm{X}^{2}(2, \quad n=49)=5.168, P=.023\right.$, Cramer's $V=.325$ ) but not between ARMS-BS and ARMS-A/B $\left(\mathrm{X}^{2}(2, n=38)=0.331, P=.565\right.$, Cramer's $V=.093$ ) for working memory. ARMS-BS patients were more frequently found in the cell with lower working memory performance compared to controls.

Three regression models were run to identify if symptomatology predicts working memory. All were non-significant. The first one included the number of cognitive BS $(F(1,36)=.004, P=.948)$, the second one included a separation of cognitive BS into cognitive disturbances and perceptual disturbances $(F(2,35)=.037, P=.964)$ and the third model included the number of cognitive BS, APS and BLIPS $(F(3,34)$ $=.226, P=.878$ ).

Because depressive symptoms are known to interfere with neurocognitive performance, we evaluated possible confounding effects following criteria suggested by Addington and colleagues. ${ }^{53}$ Comparing the ARMS-subgroups with (ARMS+D: CDSS $>6 ; n=18$ ) versus without (ARMS-D: CDSS $\leq 6$; $n=20$ ) clinically relevant depressive symptoms, a group difference in working memory performance became apparent (ARMS + D: $52.67 \pm 8.51$ vs. ARMS - D: $46.35 \pm 8.76 ; \quad T=-2.25, d f=36, P=.031$, 95\% CI [-12.01, -0.62$], d=.516)$. Regarding the global composite score no significant differences occurred (ARMS + D: $45.39 \pm 9.36$ vs. ARMS - D: $41.5 \pm 8.76$; $T=-1.40, d f=36, \quad P=0.171,95 \%$ CI $[-9.54,1.76]$, $d=321$ ).

Furthermore, we evaluated potential effects of lorazepam or former cannabis abuse. A comparison of cognitive task performance in the ARMSsubgroups with versus without anxiolytic medication $(P=.58,95 \%$ CI $[-12.54,7.16])$ as well as with versus without cannabis abuse $(P=.18,95 \%$ CI $[-1.95$, 10.04]) revealed no significant group differences.

\section{Correlation analyses}

Regarding the ARMS-group we evaluated relations of neurocognition with depressive symptoms. We found working memory significantly correlated with CDSS ( $r=0.352, P=0.030)$ as well as the PANSS single item G6 (Depression) $(\rho=0.409, P=0.011)$. Regarding the PANSS composite subscore 'Depression' a trend towards significance was shown ( $r=0.315, P=0.054)$. There were no significant correlations between working memory or global ('composite') neurocognitive functioning and other psychopathological measures (e.g. ERIraos total 
score, PANSS composite scores, scores of PANSS fivefactor model, GAF).

\section{DISCUSSION}

Patients with schizophrenia display neurocognitive deficits. ${ }^{1,4-6}$ Working memory impairment has been discussed as a potential endophenotypic marker of schizophrenia spectrum disorders ${ }^{6}$ and is therefore expected to be already present in ARMS-patients. In line with our hypothesis, a lower working memory performance was found in the ARMS-group compared to HCs. This result is consistent with a meta-analysis by Fusar-Poli and colleagues. ${ }^{34}$ Furthermore, our results indicated a significant group difference for the visuospatial but not for the verbal component of working memory. This accords findings of Wood and colleagues ${ }^{23}$ who found distinct spatial working memory deficits in UHR-patients which were even more pronounced in patients who later converted to psychosis. Similarly, a metaanalysis by Lee and Park discusses robust findings of impairment in the visuospatial domain in patients with schizophrenia. ${ }^{5}$ This result gives implications for the development of an optimized assessment of cognitive deficits in the early stages of psychosis, with a focus on visuospatial working memory functioning. There are other studies in contrast, summarized in a recent review, ${ }^{54}$ which could not find working memory deficits in similar samples of ARMS-patients with cognitive BS, APS and/or BLIPS, ${ }^{10,44,55,56}$ although the latter one nevertheless found the performance between groups to reach medium effect sizes. Results regarding visual working memory in ARMS-patients are likewise inconsistent. ${ }^{55,56}$ Importantly, though working memory performance in ARMS-patients was lower than that of controls in this present study, we did not reveal deficient scores in terms of normative $t$-values. These contradictions might be explained in the light of work by Pukrop et al. ${ }^{57}$ who found that working memory is actually a measure of three distinct functions: a general comparator function, an attentional resource allocation function, and a maximum capacity storage function. The implementation of a variety of working memory tasks which might be based on different of these underlying functions could therefore lead to diverging results.

Besides working memory impairments, our findings indicated a lower global ('composite') neurocognitive functioning and deficits in the domains of processing speed, verbal learning and problem solving in the ARMS. These results are in line with independent observations $^{14,19,20,55,56}$ and a comprehensive review ${ }^{58}$ of ARMS-patients and in turn support the sensitive characterization of the ARMS using ERIraos. Again, performance of ARMS-patients in these three neuropsychological domains was not deficient in terms of $t$-values, compared to the normative sample. ${ }^{59}$ The frequency analyses demonstrated an identical pattern. Only the problem solving performance tended to be more deficient in the ARMS-group. It was suggested that the measures of processing speed, verbal learning and verbal fluency might help to improve predictions of transitions to psychosis $^{54,55}$ and more pronounced impairment was found in patients with a first episode of psychosis. $^{48,56}$ Therefore, we reason that decreased cognitive performance in these domains is a clinically relevant feature of ARMS-patients even if scores do not indicate a clear deficit. In an attempt to explain diverging results between studies, a very important consideration is the use of varying diagnostic tools. This was the first study to examine neurocognition based on the current version of ERIraos. ${ }^{41}$ Furthermore, as the MCCB is a cognitive battery which was build up for patients with schizophrenia ${ }^{59}$ the tests might not all be sensitive enough to detect deficits in the ARMS in all domains. The same has been suggested for the WCST. $^{60}$ Differing compositions of ARMS-patients as well as the general heterogeneity within ARMS-groups between studies potentially lead to findings of more or less severe neurocognitive deficits. It seems necessary to report the proportion of patients within the separate ARMSgroups when analysing merged groups.

Up to date, inconsistent results exist about the association between neurocognitive impairment and the conversion to psychosis. ${ }^{10,16,17,23,34}$ Some studies suggested that the largest impairment occurs before psychosis onset. ${ }^{10,19}$ In our crosssectional analysis, no associations between symptomatology and working memory or global neurocognitive performance were discovered and symptomatology did not predict neurocognitive impairment. However, we examined the heterogeneous ARMS-group more closely and revealed differences in working memory and global cognitive performance between the control group and both ARMS subgroups. No differences were found between ARMS-BS and ARMS-A/B. Somewhat dissenting results were found regarding global neurocognition when the second analysis strategy was implemented. ARMS-subgroups did not differ from controls anymore. A potential reason can be found in the loss of statistical power by a categorization of the variable. More even because the variable already just represented a summarized value of different cognitive functions. 
The present findings underscore that early ARMSstages, characterized by the experience of cognitive basic symptoms, are already accompanied by similar neurocognitive impairment as patients in later stages. Findings of Koutsouleris and colleagues support this implication. The authors could differentiate ARMS-BS from healthy controls on the basis of visual working memory and verbal learning using a machine-learning procedure. ${ }^{61}$ ARMS-A/B were differentiated from healthy controls mainly by means of verbal IQ, executive functions and processing speed. Thus, very similar neurocognitive domains were identified in their study and ours. In another study, impairment was slightly more severe in patients in late ARMScompared to early ARMS-stages but without statistical significance. ${ }^{38}$ Taken together, we reason that the assessment of the whole spectrum of ARMS-symptoms, when defining ARMS-samples, is highly important in multiple ways: As Ruhrmann and colleagues showed, considering both UHRcriteria and cognitive disturbances (COGDIS) improves the sensitivity for predicting psychotic transitions. ${ }^{42}$ Likewise, another comparison of the exclusive assessment of UHR-criteria with a combination of UHR-criteria and COGDIS revealed improved sensitivity for the use of both criteria. ${ }^{62}$ As the present results emphasize, the composition of cognitive BS and UHR-criteria is also reasonable to sensitively assess cognitive impairment, even before a transition to psychosis. This is in line with a recent study showing that cognitive BS and APS in combination with a processing speed deficit turned out to be an 'optimized stratified risk assessment'. ${ }^{18}$ The detection of working memory constraints in the ARMS indicates the need for effective early therapeutic interventions, such as cognitive remediation-trainings, to improve functional outcome. $^{2}$

The comparison of the subgroups ARMS +D and ARMS - D revealed lower working memory scores in the ARMS - D group. Furthermore, our findings revealed significant positive correlations between working memory and clinical relevant depression (CDSS, PANSS), suggesting that subjects with higher depression scores might have better working memory performance. These findings were surprising, as prior research has discussed depression to go along with impaired working memory function. ${ }^{63-65}$ Other studies did not find any association. ${ }^{13,66}$ So far, heterogeneity within the merged ARMS-sample cannot be excluded and it is open to ask, whether depressive symptoms might be linked to an altered transition risk to psychosis. Also, the possibility that the ARMS $+\mathrm{D}$ group mainly consisted of patients who will develop an affective disorder should be regarded. With this in mind, the present results might support prior findings which showed that depressed patients had better global neurocognitive ${ }^{67}$ and working memory skills ${ }^{68}$ than patients with schizophrenia or ARMS-patients who later converted to psychosis. ${ }^{69}$

\section{LIMITATIONS}

This study is limited because of the crosssectional design, the mono-centric approach, the lack of psychiatric control groups and the lack of data regarding transitions to psychosis. The small sample size of the individual ARMS-subgroups might have increased beta-error and therefore might have covered possibly existing working memory differences between controls and ARMS-BS. Longitudinal studies are necessary to associate cognitive deficits with the risk for transition and to investigate the longitudinal changes of these deficits.

\section{CONCLUSION}

The early and likewise valid identification and the comprehensive characterization of ARMS-patients currently display some of the crucial topics in schizophrenia research. ${ }^{37}$ The present study is the first to focus on working memory abilities in ARMSpatients characterized by ERIraos. Working memory abilities were significantly lower in ARMS-patients compared to controls, lower in ARMS-BS compared to controls and no differences became evident between ARMS-BS and ARMS-A/B. This provides new support for the hypothesis that working memory deficits might be cognitive markers of psychosis development. ${ }^{6,70}$ The results further suggest that not working memory alone but rather a combination of cognitive malfunctions, most importantly problem solving, processing speed and verbal learning, contribute to cognitive impairment in the ARMS. Forthcoming studies on neurocognition in the ARMS should reflect the entire spectrum of symptoms including cognitive BS concepts. Longitudinal studies should investigate if a combination of cognitive BS assessed with ERIraos and the identified cognitive domains might allow for an early estimation of the transition risk and might include other psychiatric control groups to explore the specificity of these deficits to psychosis. Not least because of the cognitive impairment, therapeutic interventions should already be implemented in the early ARMS 
to improve the symptomatology and circumvent possible deteriorations of existing constraints.

\section{ACKNOWLEDGEMENTS}

M.Z., A.M.-L. and P.K. were funded by the Deutsche Forschungsgesellschaft (DFG, http:// www.dfg.de, projects ZI1253/3-1, ZI1253/3-2, KI 576/14-2, ME 1591/6-2). Sarah Eisenacher was supported by a Grant of Heidelberg University (Landesgraduiertenförderungsgesetz) and Daniela Mier by the Olympia-Morata Program. The funders had no role in study design, data collection and analysis, decision to publish or preparation of the manuscript.

\section{REFERENCES}

1. Keefe RS, Bilder RM, Harvey PD, et al. Baseline neurocognitive deficits in the CATIE schizophrenia trial. Neuropsychopharmacol: Official Publ Am College Neuropsychopharmacol 2006; 31 (9): 2033-46.

2. Fett $A K$, Viechtbauer W, Dominguez M-G, Penn DL, van Os J, Krabbendam $\mathrm{L}$. The relationship between neurocognition and social cognition with functional outcomes in schizophrenia: a meta-analysis. Neurosci Biobehav Rev 2011; 35 (3): 573-88.

3. McGurk SR, Twamley EW, Sitzer DI, McHugo GJ, Mueser KT. A meta-analysis of cognitive remediation in schizophrenia. Am J Psych 2007; 164 (12): 1791-802.

4. Nuechterlein KH, Barch DM, Gold JM, Goldberg TE, Green MF, Heaton RK. Identification of separable cognitive factors in schizophrenia. Schizophr Res 2004; 72 (1): 29-39.

5. Lee J, Park S. Working memory impairments in schizophrenia: a meta-analysis. J Abnorm Psychol 2005; 114 (4): 599-611.

6. Park S, Gooding DC. Working memory impairment as an endophenotypic marker of a schizophrenia diathesis. SchizophrResCogn 2014; 1 (3): 127-36.

7. Forbes NF, Carrick LA, McIntosh AM, Lawrie SM. Working memory in schizophrenia: a meta-analysis. Psychol Med 2009; 39 (6): 889-905.

8. Snitz BE, Macdonald AWI, Carter CS. Cognitive deficits in unaffected first-degree relatives of schizophrenia patients: a meta-analytic review of putative endophenotypes. Schizophr Bull 2006; 32 (1): 179-94.

9. Zanello A, Curtis L, Badan BM, Merlo MC. Working memory impairments in first-episode psychosis and chronic schizophrenia. Psychiatry Res 2009; 165 (1-2): 10-8.

10. Becker HE, Nieman DH, Wiltink $S$, et al. Neurocognitive functioning before and after the first psychotic episode: does psychosis result in cognitive deterioration? Psychol Med 2010; 40 (10): 1599-606.

11. Brewer WJ, Wood SJ, Phillips LJ, et al. Generalized and specific cognitive performance in clinical high-risk cohorts: a review highlighting potential vulnerability markers for psychosis. Schizophr Bull 2006; 32 (3): 538-55.

12. de Paula AL, Hallak JE, Maia-de-Oliveira JP, Bressan RA, Machado-de-Sousa JP. Cognition in at-risk mental states for psychosis. Neurosci Biobehav Rev 2015; 57: 199-208.

13. Frommann I, Pukrop R, Brinkmeyer J, et al. Neuropsychological profiles in different at-risk states of psychosis: executive control impairment in the early - and additional memory dysfunction in the late--prodromal state. Schizophr Bull 2011; 37 (4): 861-73.

14. Jahshan C, Heaton RK, Golshan S, Cadenhead KS. Course of neurocognitive deficits in the prodrome and first episode of schizophrenia. Neuropsychology 2010; 24 (1): 109-20.

15. Mourik K, Decrescenzo P, Brucato G, Gill KE. Various neurocognitive deficits and conversion risk in individuals at clinical high risk for psychosis. Early Interv Psychiatry 2015. DOI: 10.1111/eip.12296 Epub ahead of print.

16. Seidman LJ, Giuliano AJ, Meyer EC, et al. Neuropsychology of the prodrome to psychosis in the napls consortium: relationship to family history and conversion to psychosis. Arch Gen Psychiatry 2010; 67 (6): 578-88.

17. Woodberry KA, Seidman LJ, Giuliano AJ, Verdi MB, Cook WL, McFarlane WR. Neuropsychological profiles in individuals at clinical high risk for psychosis: relationship to psychosis and intelligence. Schizophr Res 2010; 123 (2-3): 188-98.

18. Michel C, Ruhrmann S, Schimmelmann BG, Klosterkötter J, Schultze-Lutter F. A stratified model for psychosis prediction in clinical practice. Schizophr Bull 2014; 40 (6): 1533-42.

19. Bora E, Murray R. Meta-analysis of cognitive deficits in ultrahigh risk to psychosis and first-episode psychosis: do the cognitive deficits progress over, or after, the onset of psychosis? Schizophr Bull 2014; 40 (4): 744-55.

20. De Herdt A, Wampers $M$, Vancampfort $D$, et al. Neurocognition in clinical high risk young adults who did or did not convert to a first schizophrenic psychosis: a metaanalysis. Schizophr Res 2013; 149 (1-3): 48-55.

21. Goghari VM, Brett C, Tabraham P, et al. Spatial working memory ability in individuals at ultra high risk for psychosis. $J$ Psychiatr Res 2014; 50: 100-5.

22. Pflueger MO, Gschwandtner U, Stieglitz RD, Riecher-Rossler A. Neuropsychological deficits in individuals with an at risk mental state for psychosis-working memory as a potential trait marker. Schizophr Res 2007; 97 (1-3): 14-24.

23. Wood SJ, Pantelis C, Proffitt T, et al. Spatial working memory ability is a marker of risk-for-psychosis. Psychol Med 2003; 33 (7): 1239-47.

24. Simon AE, Cattapan-Ludewig K, Zmilacher S, et al. Cognitive functioning in the schizophrenia prodrome. Schizophr Bull 2007; 33 (3): 761-71.

25. Simon $A E$, Gradel $M$, Cattapan-Ludewig $K$, et al. Cognitive functioning in at-risk mental states for psychosis and 2-year clinical outcome. Schizophr Res 2012; 142 (1-3): 108-15.

26. Kelleher I, Murtagh A, Clarke MC, Murphy J, Rawdon C, Cannon M. Neurocognitive performance of a communitybased sample of young people at putative ultra high risk for psychosis: support for the processing speed hypothesis. Cogn Neuropsychiatry 2013; 18 (1-2): 9-25.

27. Niarchou M, Zammit S, Walters J, Lewis G, Owen MJ, van den Bree MB. Defective processing speed and nonclinical psychotic experiences in children: longitudinal analyses in a large birth cohort. Am J Psychiatry 2013; 170 (5): 550-7.

28. Cannon M, Moffitt TE, Caspi A, Murray RM, Harrington $H$, Poulton R. Neuropsychological performance at the age of 13 years and adult schizophreniform disorder: prospective birth cohort study. Br J Psychiatry 2006; 189: 463-4.

29. Miller TJ, McGlashan TH, Woods SW, et al. Symptom assessment in schizophrenic prodromal states. Psychiatry $Q$ 1999; 70 (4): 273-87.

30. Yung AR, Phillips LJ, McGorry PD, et al. Prediction of psychosis. A step towards indicated prevention of schizophrenia. $\mathrm{Br} J$ Psychiatry Suppl 1998; 172 (33): 14-20.

31. Miller TJ, McGlashan TH, Rosen JL, et al. Prodromal assessment with the structured interview for prodromal syndromes and the scale of prodromal symptoms: predictive validity, interrater reliability, and training to reliability. Schizophr Bull 2003; 29 (4): 703-15. 
32. Yung A, Yuen HP, McGorry PD, et al. Mapping the onset of psychosis: the comprehensive assessment of at-risk menta states. Aust New Zealand J Psych 2005; 39 (11-12): 964-71.

33. Addington J, Barbato $M$. The role of cognitive functioning in the outcome of those at clinical high risk for developing psychosis. Epidemiol Psych Sci 2012; 21 (04): 335-42.

34. Fusar-Poli P, Deste G, Smieskova R, et al. Cognitive functioning in prodromal psychosis: a meta-analysis. Arch Gen Psychiatry 2012; 69 (6): 562-71.

35. Huber G, Gross G. The concept of basic symptoms in schizophrenic and schizoaffective psychoses. Recenti Prog Med 1989; 80 (12): 646-52.

36. Ebel H, Gross G, Klosterkotter J, Huber G. Basic symptoms in schizophrenic and affective psychoses. Psychopathology 1989; 22 (4): 224-32.

37. Schultze-Lutter F, Ruhrmann S, Berning J, Maier W, Klosterkotter J. Basic symptoms and ultrahigh risk criteria: symptom development in the initial prodromal state. Schizophr Bull 2010; 36 (1): 182-91.

38. Schultze-Lutter F, Addington J, Ruhrmann S, Klosterkoetter J. Schizophrenia Proneness Instrument-Adult Version. Rome: Giovanni Fioriti Editore, 2007.

39. Hafner H, Bechdolf A, Klosterkotter J, Maurer K. PsychosenFrüherkennung und Frühintervention, Der Praxisleitfaden. Stuttgart: Schattauer, 2012.

40. Maurer K, Zink M, Rausch F, Häfner $H$. The early recognition inventory ERIraos assesses the entire spectrum of symptoms through the course of an at-risk mental state. Early Interven Psychiatry 2016. DOI: 10.1111/eip.12305. [Epub ahead of print].

41. Rausch F, Eifler S, Esser A, et al. The Early Recognition Inventory ERIraos detects at risk mental states of psychosis with high sensitivity. Compr Psychiatry 2013; 54 (7): 1068-76.

42. Ruhrmann S, Schultze-Lutter F, Salokangas RK, et al. Prediction of psychosis in adolescents and young adults at high risk: results from the prospective European prediction of psychosis study. Arch Gen Psychiatry 2010; 67 (3): 241-51.

43. Becker HE, Nieman DH, Dingemans PM, van de Fliert JR, de Haan L, Linszen DH. Verbal fluency as a possible predictor for psychosis. Eur Psychiatry 2010; 25 (2): 105-10.

44. Ozgurdal S, Littmann E, Hauser $M$, et al. Neurocognitive performances in participants of at-risk mental state for schizophrenia and in first-episode patients. J Clin Exp Neuropsychol 2009; 31 (4): 392-401.

45. Kay SR, Fiszbein A, Opler LA. The positive and negative syndrome scale (PANSS) for schizophrenia. Schizophr Bull 1987; 13 (2): 261-76.

46. van der Gaag M, Hoffman T, Remijsen M, et al. The five-factor model of the Positive and Negative Syndrome Scale II: a tenfold cross-validation of a revised model. Schizophr Res 2006; 85 (1-3): 280-7.

47. Nuechterlein KH, Green MF, Kern RS, et al. The MATRICS Consensus Cognitive Battery, part 1: test selection, reliability, and validity. Am J Psych 2008; 165 (2): 203-13.

48. Eastvold AD, Heaton RK, Cadenhead KS. Neurocognitive deficits in the (putative) prodrome and first episode of psychosis. Schizophr Res 2007; 93 (1-3): 266-77.

49. Faul F, Erdfelder E, Lang AG, Buchner A. G*power 3: a flexible statistical power analysis program for the social, behavioral, and biomedical sciences. Behav Res Methods 2007; 39: 175-91.

50. Kern RS, Gold JM, Dickinson D, et al. The MCCB impairment profile for schizophrenia outpatients: results from the MATRICS psychometric and standardization study. Schizophr Res 2011; 126 (1-3): 124-31.

51. Lunney $\mathrm{GH}$. Using analysis of variance with a dichotomous dependent variable: an empirical study. J Ed Meas 1970; 7 (4): 263-9.

52. Donaldson TS. Robustness of the F-test to errors of both kinds and the correlation between the numerator and denominator of the F-ratio. J Am Stat Assoc 1968; 63 (322): 660-76.
53. Addington D, Addington J, Maticka-Tyndale E. Assessing depression in schizophrenia: the Calgary Depression Scale. British J Psych 1993; 163 (Suppl 22): 39-44.

54. Pukrop R, Ruhrmann S. Neurocognitive indicators of high-risk states for psychosis. In: Fusar-Poli P, Borgwardt S, McGuire P, eds. Vulnerability to Psychosis. From Neuroscience to Psychopathology. New York, NY: Psychology Press, 2012.

55. Schultze-Lutter F, Ruhrmann S, Heinz P, et al. Relationship between subjective and objective cognitive function in the early and late prodrome. Br J Psychiatry 2007; 191 (51): s43-s51.

56. Pukrop $\mathrm{R}$, Schultze-Lutter $\mathrm{F}$, Ruhrmann $\mathrm{S}$, et al. Neurocognitive functioning in subjects at risk for a first episode of psychosis compared with first- and multipleepisode schizophrenia. J Clin Exp Neuropsychol 2006; 28 (8): 1388-407.

57. Pukrop R, Matuschek E, Ruhrmann S, et al. Dimensions of working memory dysfunction in schizophrenia. Schizophr Res 2003; 62 (3): 259-68.

58. Pukrop R, Klosterkotter J. Neurocognitive indicators of clinical high-risk states for psychosis: a critical review of the evidence. Neurotox Res 2010; 18 (3-4): 272-86.

59. Nuechterlein $\mathrm{KH}$, Green MF, eds. MCCB MATRICS Consensus Cognitive Battery. Los Angeles: The Regents of the University of California, 2006.

60. Goldstein G, Beers SR, Shemansky WJ. Neuropsychological differences between schizophrenic patients with heterogeneous Wisconsin Card Sorting Test performance. Schizophr Res 1996; 21 (1): 13-8.

61. Koutsouleris N, Davatzikos C, Bottlender R, et al. Early recognition and disease prediction in the at-risk mental states for psychosis using neurocognitive pattern classification. Schizophr Bull 2012; 38 (6): 1200-15.

62. Schultze-Lutter F, Klosterkotter J, Ruhrmann S. Improving the clinical prediction of psychosis by combining ultra-high risk criteria and cognitive basic symptoms. Schizophr Res 2014; 154 (1-3): 100-6.

63. Baune BT, Fuhr M, Air T, Hering C. Neuropsychological functioning in adolescents and young adults with major depressive disorder-a review. Psychiatry Res 2014; 218 (3): 261-71.

64. Möser C, Krieg JC, Zihl J, Lautenbacher S. Attention and memory deficits in schizophrenia: the role of symptoms of depression. Cogn Behav Neurol 2006; 19 (3): 150-6.

65. Riedel M, Schennach-Wolf R, Musil R, et al. Neurocognition and its influencing factors in the treatment of schizophrenia -effects of aripiprazole, olanzapine, quetiapine and risperidone. Human Psychopharmacol: Clin Exp 2010; 25 (2): 116-25.

66. Metzler S, Dvorsky D, Wyss C, et al. Neurocognitive profiles in help-seeking individuals: comparison of risk for psychosis and bipolar disorder criteria. Psychol Med 2014; 44 (16): 3543-55.

67. Reichenberg A, Harvey PD, Bowie CR, et al. Neuropsychological function and dysfunction in schizophrenia and psychotic affective disorders. Schizophr Bull 2009; 35 (5): 1022-9.

68. Egeland J, Rund BR, Sundet $\mathrm{K}$, et al. Attention profile in schizophrenia compared with depression: differential effects of processing speed, selective attention and vigilance. Acta Psychiatr Scand 2003; 108 (4): 276-84.

69. Schulze C, Zimmermann R, Gschwandtner U, et al. Can cognitive deficits facilitate differential diagnosis between atrisk mental state for psychosis and depressive disorders? Early Interv Psychiatry 2013; 7 (4): 381-90.

70. Fusar-Poli P, Broome MR, Matthiasson P, et al. Prefrontal function at presentation directly related to clinical outcome in people at ultrahigh risk of psychosis. Schizophr Bull 2011; 37 (1): 189-98. 